\title{
Mass dependent Evolution of Field Early-Type Galaxies Since $z=1$
}

\author{
Alexander Fritz, Inger Jørgensen, \& Ricardo P. Schiavon \\ Gemini Observatory, 670 N. A'ohoku Place, Hilo, HI 96720, USA \\ e-mail: afritz@gemini.edu
}

\begin{abstract}
We present the Fundamental Plane (FP) of field early-type galaxies at $0.5<z<1.0$. Our project is a continuation of our efforts to understand the formation and evolution of earlytype galaxies in different environments. The target galaxies were selected from the comprehensive and homogeneous data set of the Gemini/HST Galaxy Cluster Project. The distant field earlytype galaxies follow a steeper FP relation compared to the local FP. The change in the slope of the FP can be interpreted as a mass-dependent evolution. Similar results have been found for cluster early-type galaxies in high redshift galaxy clusters at $0.8<z<1$. Therefore, the slope change of the FP appears to be independent of the environment of the galaxies.
\end{abstract}

Keywords. cosmology: observations - galaxies: evolution - galaxies: structure - galaxies: elliptical and lenticular, $\mathrm{cD}$ - galaxies: fundamental parameters - galaxies: stellar content

\section{Introduction}

Early-type galaxies obey a tight linear relation in 3-D log-space, the Fundamental Plane (FP; Djorgovski \& Davis 1987; Dressler et al. 1987), defined by their size $r_{\mathrm{e}}$, average surface brightness $\left\langle I_{\mathrm{e}}\right\rangle$ and stellar velocity dispersion $(\sigma)$. Via a study of the FP constraints on the formation epoch and evolution of early-type galaxies are possible.

Over the past twenty years a significant effort has been devoted to understand the physical processes involved in this empiric relationship in the nearby universe. Previous works of field early-type galaxies at higher redshift up to $z \sim 1.2$ found evidence for a mass dependent evolution (e.g., Treu et al. 2005; di Serego Alighieri et al. 2006). Recently, Fritz et al. (2009a) detected recent star formation in less-massive field early-type galaxies up to $z \sim 0.8$. This rejuvenation accounts for $5-10 \%$ in the total stellar mass budget of these galaxies, possible triggered through AGN feedback.

\section{Observational Data}

Target galaxies were selected from the Gemini/HST Galaxy Cluster Project. This project is an extensive observational campaign to investigate the formation and evolution of distant galaxies in rich galaxy clusters from $z=1$ to the present-day (Jørgensen et al. $2005)$. For 15 massive, $X$-ray luminous galaxy clusters $\left(L_{X}(0.1-2.4 \mathrm{keV}) \leqslant 2 \times 10^{44}\right.$ $\operatorname{erg~} \mathrm{s}^{-1}$ ) from $z=0.1$ to 1 , intermediate-resolution Gemini/GMOS spectroscopy and HST/ACS or WFPC2 imaging were obtained. The MOS masks included primarily expected cluster members, while vacant space was filled with slits for objects that have similar brightnesses but slightly bluer or redder colours than expected for early-type cluster members. Out of three cluster fields, RXJ0152.7-1357, RXJ1226.9+3332 and RXJ1415.1+3612, we have extracted 20 galaxies with early-type morphology and $0.5<$ $z<1(\langle z\rangle=0.74)$ which are used in the following. The high $S / N$ and intermediate resolution galaxy spectra $\left(\langle S / N\rangle \sim 25 \stackrel{\circ}{A}_{\text {rest }}^{-1}\right)$ allows us to study in detail the internal 

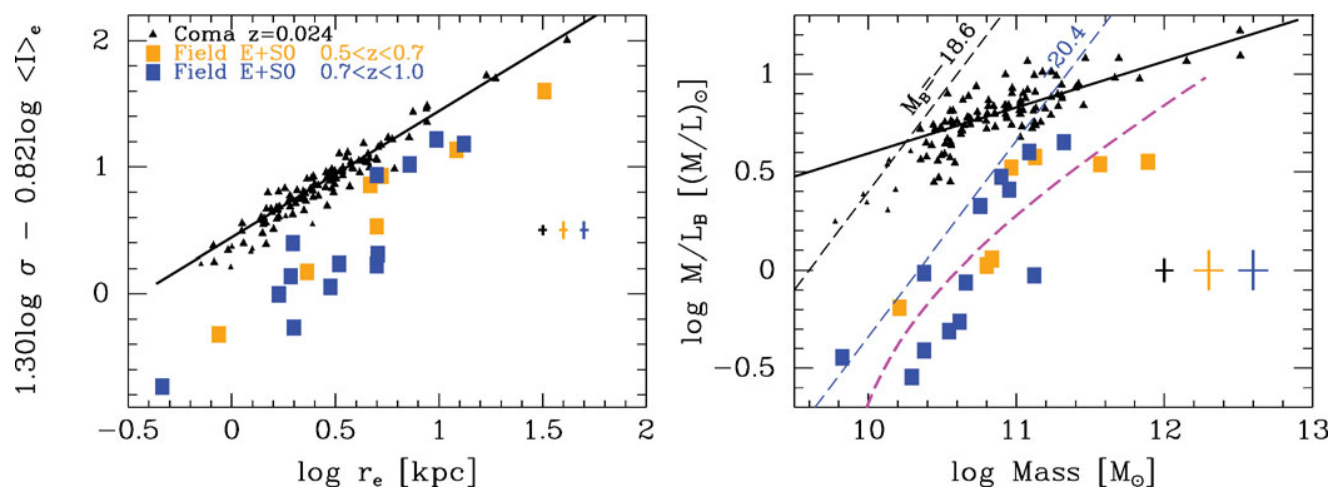

Figure 1. Fundamental Plane (FP) of field early-type galaxies in two redshift bins $0.5<z<0.7$ (orange) and $0.7<z<1.0$ (blue). Left: Edge-on projection of FP. The Coma cluster is used as a local reference (black triangles, solid line, Jørgensen 1999). Right: $M / L-M$ plane. Dashed lines indicate the magnitude limits of Coma (black) and the distant field galaxies (large symbols). SSP model predictions are indicated as the dashed magenta line (Thomas et al. 2005).

kinematics and stellar populations of the galaxies. Using deep HST/ACS imaging, we fit model profiles to the galaxies to derive the effective parameters (Chiboucas et al. 2009).

\section{The FP of Field Early-Type Galaxies up to $z=1$}

Fig. 1 shows the FP for field early-type galaxies (squares) in rest-frame Johnson $B$ band, compared to 116 early-type galaxies in the Coma cluster (triangles). The slope of the FP for field galaxies at high redshift is steeper than that for their local counterparts. We interpret the slope difference between the distant and local relation as a mass-dependent evolution. Less-massive field galaxies $\left(M \leqslant 8 \times 10^{10} M_{\odot}\right)$ evolve faster than their more massive counterparts. The $M / L$ evolution with $z$ is under investigation. Similar results have been found for galaxy clusters at $0.8<z<1.0$ (Jørgensen et al. 2006, 2007; Fritz et al. 2009b). This suggest that environmental effects play only a minor role on the evolution of early-type galaxies. A combined analysis of the FP and absorption line strengths for the field galaxies will be presented in Fritz et al. (2009c, in prep).

AF and IJ acknowledge support from grant HST-GO-10826.01 from STScI. Based on observations obtained at the Gemini Observatory, operated by AURA, Inc., under cooperative agreement with the NSF, on behalf the NSF, STFC (UK), NRC (Canada), CONICYT (Chile), ARC (Australia), CNPq (Brazil), and SECYT (Argentina).

\section{References}

Chiboucas, K., Barr, J., Flint, K., et al. 2009, ApJS, 184, 271

di Serego Alighieri, S., Lanzoni, B., \& Jørgensen, I. 2006, ApJ, 652, L145

Djorgovski, S. \& Davis, M. 1987, ApJ, 313, 59

Dressler, A., et al. 1987, ApJ, 313, 42

Fritz, A., Böhm, A., \& Ziegler, B. L. 2009a, MNRAS, 393, 1467

Fritz, A., Jørgensen, I., Schiavon, R. P., \& Chiboucas, K. 2009b, AN, in press (arXiv:0910.4007) Jørgensen, I. 1999, MNRAS, 306, 607

Jørgensen, I., Bergmann, M., Davies, R., et al. 2005, AJ, 129, 1249

Jørgensen, I., et al. 2006, ApJ, 639, L9

Jørgensen, I., et al. 2007, ApJ, 654, L179

Thomas, D., et al. 2005, ApJ, 621, 673

Treu, T., et al. 2005, ApJ, 633, 174 